\title{
Dimensionality reduction for discrimination: removal of common structures with iSTAC
}

\author{
Graham I Cummins*, Alexander G Dimitrov \\ From Nineteenth Annual Computational Neuroscience Meeting: CNS*2010 \\ San Antonio, TX, USA. 24-30 July 2010
}

Many neuroscience experiments, particularly those directed at exploring the relationship between sensory input and neural responses through various uses of a response conditioned stimulus ensemble (RCSE) use gaussian white noise (GWN) stimuli. The use of GWN allows simplification of the analysis, since this is some sort of comparison between the RCSE and the distribution of undconditioned or raw stimuli (The UCSE). In the GWN case, the UCSE is known, and has the simplifying properties of being uncorrelated and normally distributed. Ths is useful, but using the theoretical properties of GWN inputs too aggressively can lead to errors stemming from the fact that real inputs are only approximately Gaussian and white. In particular, all stimuli used in computer driven experiments are band limited. Even a "purely" white stimulus will be band limited due to the constraints of being discretely sampled and finitely long, but in a typical experiment, explicit band limits are applied by the experimenters, which are more restrictive than the limits due to sampling.
Band limited stimuli have an auto-correlation structure, which can be quite substantial for band widths of ethological interest. For example, the cercal sensory system of crickets detects air movements at frequencies typically less than $200 \mathrm{~Hz}$. The covariance matrix of a $5-200 \mathrm{~Hz}$ band-limited signal is shown in Figure 1A. We use a model cell to select a RCSE subset of that stimulus, presenting its mean and covariance in panels $1 \mathrm{~B}$ and $1 \mathrm{C}$. It is obvious from looking at $1 \mathrm{C}$ that a large portion of its structure is determined by the covariance of the UCSE in 1A. This affects the quality of models built to explain the structure of the RCSE and provide insight into neural processing.

We designed an approach with which to reduce the effects of nontrivial UCSE structure in the analysis of the RCSE. The main goal of that approach was to remove structures that were common in both sets and thus did not contribute to discriminating between the conditioned and unconditioned stimulus. Results of applying this approach to the set described above can be
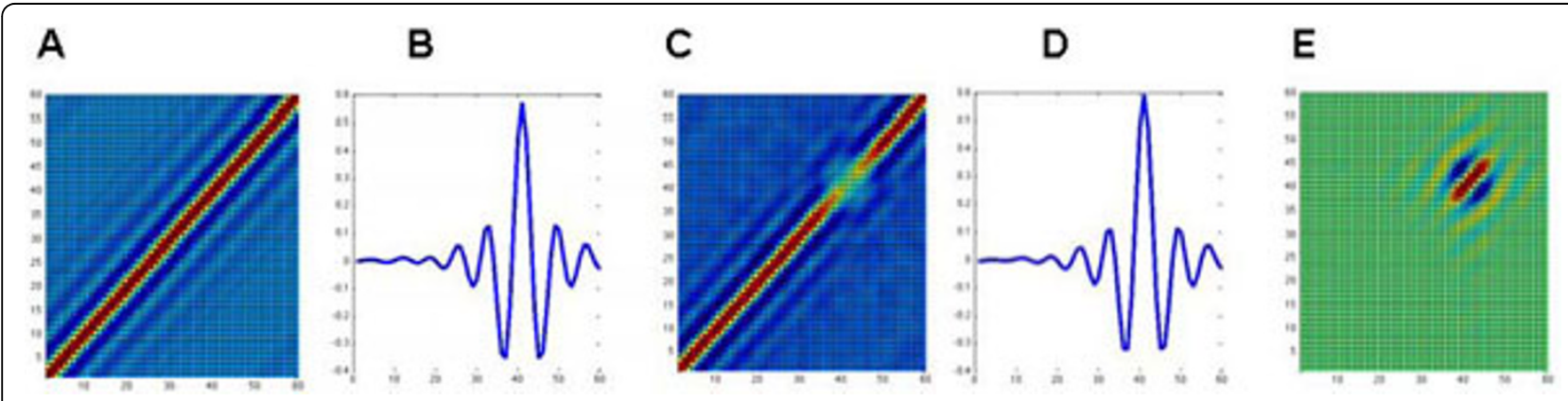

Figure $1 \mathrm{~A}$. Structure of the unconditional covariance. B. Conditional mean and C. Covariance of a model cell. D. Conditional mean and E. Covariance after iSTAC compression.

\footnotetext{
* Correspondence: alex@cns.montana.edu

Center for Computational Biology, Montana State University, Bozeman, MT,
} USA 
seen in Figure 1D and 1E The model mean shown in 1D is very similar to the mean obtained before the correction. However, the covariance seen in $1 \mathrm{E}$ shows that the method successfully removes most of the common structure, previously observed in $1 \mathrm{~A}$ and $1 \mathrm{C}$ and retains and enhances covariance structure that helps in discriminating between the RCSE and the UCSE.

We employed the iSTAC method [1] for that purpose. In its original use, iSTAC was designed to find a small subspace that maintains as much as possible of the Kullback-Leibler (KL) divergence between two distributions. For the dimensionality reduction application here, we used iSTAC to discard as much as possible of the common signal structure discussed earlier, by removing the subspace that does not affect the KL divergence much. In Figure 1 we show the results after removing about half of the dimensions, while still retaining $90 \%$ of the overall variance of the stimulus.

Published: 20 July 2010

\section{Reference}

1. Pillow JW, Simoncelli EP: Dimensionality reduction in neural models: An information-theoretic generalization of spike-triggered average and covariance analysis. J Vis 2006, 6:414-428.

doi:10.1186/1471-2202-11-S1-P176

Cite this article as: Cummins and Dimitrov: Dimensionality reduction for discrimination: removal of common structures with iSTAC. BMC

Neuroscience 2010 11(Suppl 1):P176.
Submit your next manuscript to BioMed Central and take full advantage of:

- Convenient online submission

- Thorough peer review

- No space constraints or color figure charges

- Immediate publication on acceptance

- Inclusion in PubMed, CAS, Scopus and Google Scholar

- Research which is freely available for redistribution

Submit your manuscript at www.biomedcentral.com/submit 